\title{
Nonperturbative electron-ion scattering theory incorporating the Møller interaction
}

\author{
C. Bostock ${ }^{1}$, D. V. Fursa, I. Bray \\ ARC Centre for Antimatter-Matter Studies, Curtin University of Technology, \\ GPO Box U1987, Perth, WA 6845, Australia
}

\begin{abstract}
Synopsis We present the first calculations that investigate the effects of both the Møller interaction and closecoupling in the calculation of electron-impact excitation cross sections. Electron scattering from $\mathrm{U}^{91+}$ is used as a test case. The RCCC method is nonperturbative and we emphasise the restrictions and subsequent limitations associated with employing the Møller interaction in the RCCC method.
\end{abstract}

The first order relativistic distorted wave calculations of electron-impact excitation of highly charged ions by Fontes [1] have shown that the Generalized Breit interaction (equivalently the Møller interaction) can significantly affect cross sections by up to $50 \%$ in comparison to Coulombonly interaction calculations. An important consideration that has recently been investigated is the incorporation of the Møller interaction in close-coupling scattering calculations [2].

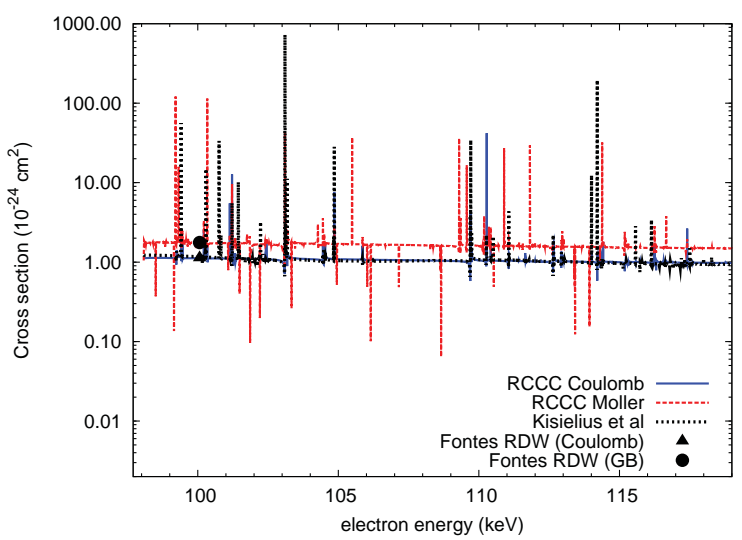

Figure 1. $1 s_{1 / 2} \rightarrow 2 s_{1 / 2}$ electron-impact excitation RCCC-calculated cross section (solid blue line Coulomb-interaction, dashed red line Møllerinteraction) compared with R-matrix 9 state calculation of [3] (dotted black line) and the first order relativistic distorted wave calculations of [1] (black triangle Coulomb-interaction, black circle GB-interaction).
Close-coupling (nonperturbative) calculations are required to resolve resonance features in electron impact excitation cross sections; resonant features are absent in first order perturbative calculations and yet such resonances can have a significant contribution to effective collision strengths obtained by integrating over Maxwellian distributions of electron velocities corresponding to temperatures found in astrophysical and torrential fusion plasmas. The issues surrounding the use of the Møller interaction in a non-perturbative formalism are discussed in detail in [2], and also presented in the same work are excitation cross sections for electron impact on hydrogenlike uranium. We have found that the effects of close-coupling are simply to introduce sharp resonances on top of an overall background cross section, the magnitude of which can be obtained with a first order calculation. Therefore the dominant rise in the cross section due to the Møller interaction can be obtained with a first order calculation.

\section{References}

[1] Fontes C J, Sampson D H and Zhang H L 1993 Phys. Rev. A 47 1009-1022

[2] Bostock C J, Fursa D V and Bray I 2012 Phys. Rev. A 86(4) 042709

[3] Kisielius R, Berrington K A and Norrington P H 1995 Journal of Physics B: Atomic, Molecular and Optical Physics 282459

\footnotetext{
${ }^{1}$ E-mail: c.bostock@curtin.edu.au
} 